\title{
The 3D Structure of the Galactic Bulge from the MACHO Red Clump Stars
}

\author{
R. Fux and T. Axelrod \\ Research School of Astronomy and Astrophysics, Mount Stromlo \\ Observatory, Canberra ACT 2611, Australia \\ P. Popowski
}

Lawrence Livermore National Laboratory, CA 94551, USA

\section{Introduction}

Many evidences demonstrate that the Milky Way is a barred galaxy with the near end of the bar pointing in the first Galactic quadrant (e.g. Gerhard 1999 for a review), thus offering an exceptional chance to study the detailed threedimensional structure and dynamics of a real bar. One of the best tracer of the Galactic bulge/bar are the red clump stars: they are very numerous, sufficiently bright to be detected throughout the bulge, and their absolute I-magnitude has an intrinsic dispersion of only 0.2 magnitude, with a mean value almost independent of colour and metallicity (Paczyński \& Stanek 1998, Udalski 2000). Here we outline an ongoing project aiming to recover the 3D bulge stellar distribution as primarily traced by these stars, as in Stanek et al. (1997), but in a non-parametric way and from the $V R$ database of the MACHO experiment within an almost fully observed $\sim 8^{\circ} \times 8^{\circ}$ bulge area centred on $(\ell, b) \approx\left(4^{\circ},-6^{\circ}\right)$ (e.g. Alcock et al. 1997), soon complemented with $I$-band photometry.

\section{Deconvolution methods}

Because it is impossible to distinguish individual red clump stars from the other giants and from the foreground main sequence stars in a colour-apparent magnitude diagram, we will adopt a statistical approach based on the stars within the red clump colour range and involving their associated luminosity function. Assuming extinction corrected magnitudes, the fundamental equation relating the observed number of stars $A(m) d m$ per unit solid angle with apparent magnitude between $m$ and $m+d m$ and the stellar number density $\nu(s)$ as a function of distance $s$ along the line of sight is the convolution integral:

$$
A(m)=\int_{-\infty}^{\infty} \Sigma(x) \phi(m-x) d x .
$$

where $x$ is the distance modulus, $\Sigma(x) \equiv s^{3} \nu(s) /(5 \log e)$ and $\phi(M)$ is the normalised absolute magnitude function. The latter function is not known a priori, but may for instance be constrained imposing that the recovered stellar distribution is symmetric relative to the Galactic centre at $\ell=0$. 
Two deconvolution methods will be considered and the results compared to each other. First, a useful expression of the solution to Eq. (1), involving the Fourier transform of $A(m)$ and $\phi(M)$, is obtained via the convolution theorem. It has the advantage that the contribution of the high frequencies related to statistical noise and rendering the numerical deconvolution particularly unstable can be easily filtered out, and shows that the physically meaningful $x$-resolution of the recovered $\Sigma(x)$ is essentially determined by the narrowest significant peak in $\phi(M)$, which is where the red clump peak plays a crucial role. The other method is based on the iterative Richardson-Lucy deprojection algorithm (e.g. Appendix C in Binney \& Merrifield 1998), which has the advantage to ensure a non-negative solution everywhere along the line of sight. This method has been successfully tested using the particle distribution in realistic $N$-body models of the Milky Way (e.g. Fux 1999).

The photometry may not be deep enough to be complete down to the main sequence, especially in the high extinction fields. However, in the red clump colour range, the apparent magnitude range of giants within a distance of $4 \mathrm{kpc}$ from the Galactic centre will be contaminated only by a small fraction of foreground main sequence stars, so that truncating $A(m)$ near the upper limit of this magnitude range and ignoring the main sequence in $\phi(M)$ still yields a good estimate of the true bulge stellar distribution.

\section{Dealing with extinction}

The colour selection of stars and Eq. (1) require the determination of dereddened apparent magnitudes. We will consider two approaches. In the first one, we will use a variant of the method introduced by Woźniak \& Stanek (1996) to build an extinction map of the surveyed sky area. From this map we will get dereddened colour indices $(V-I)_{0}$ and apparent magnitudes $I_{0}$ which will be used for the colour section of stars and as the variable $m$ respectively.

In the second approach, assuming a reddening law and taking full advantage of the combined $V R I$ photometry, we will work with extinction-free magnitudes (like $V_{V-I}$ in Stanek et al. 1997) and colour indices instead of the $I_{0}$ versus $(V-I)_{0}$ diagram, i.e. selecting the stars via their extinction-free colour index and expressing the stellar statistics in the extinction-free magnitude.

\section{References}

Alcock C., Allsman R.A., Alves D. et al. 1997, ApJ 479, 119

Binney J., Merrifield M. 1998. Galactic Astronomy, Princeton University Press Fux R. 1999, A\&A 345, 787

Gerhard O.E. 1999. In: Merritt D., Sellwood J.A., Valluri M. (eds.) Galaxy Dynamics. ASP Conf. Series 182, 307

Paczyński B., Stanek K.Z. 1998, ApJ 494, L219

Stanek K.Z., Udalski A., Szymański M. et al. 1997, ApJ 477, 163

Udalski A. 2000, ApJ 531, L25

Woźniak P.R., Stanek K.Z. 1996, ApJ 464, 233 